Europhys. Lett., 73 (4), pp. 513-519 (2006)

DOI: $10.1209 / \mathrm{epl} / \mathrm{i} 2005-10430-6$

\title{
Driven binary mixtures: Clustering and giant diffusion
}

\author{
F. MARChesoni ${ }^{1}$, S. SAVEL'Ev ${ }^{2,3}$ and F. Nori ${ }^{2,4}$ \\ 1 Dipartimento di Fisica, Università di Camerino, I-62032 Camerino, Italy \\ 2 Frontier Research System, The Institute of Physical and Chemical Research (RIKEN) \\ Wako-shi, Saitama, 351-0198, Japan \\ 3 Department of Physics, Loughborough University - Loughborough LE11 3TU, UK \\ 4 Center for Theoretical Physics, Department of Physics, University of Michigan \\ Ann Arbor, MI 48109-1040, USA
}

received 24 November 2005; accepted in final form 20 December 2005

published online 6 January 2006

PACS. 05.60.-k - Transport processes.

PACS. 05.40.-a - Fluctuation phenomena, random processes, noise, and Brownian motion.

PACS. 74.40.+k - Fluctuations (noise, chaos, nonequilibrium superconductivity, localization, etc.).

\begin{abstract}
We study noise-assisted transport in a binary mixture of overdamped interacting particles. As one species (termed "active") is subject to a weak dc drive, the other one (termed "passive") can be dragged along due to the clustering of particles of both species. On increasing the external drive, clusters of different size fragment at different thresholds that depend on the mixture temperature, the inter-particle interaction strength, and the densities of both species of particles. Moreover, normal self-diffusion of both species at the fragmentation thresholds undergoes a giant enhancement simultaneous with a markedly negative cross-diffusion coefficient.
\end{abstract}

Ongoing experiments on the transport of two or more ion species through an ion channel [1] and the diffusion of adsorbants of different sizes in zeolite pores [2] raise the question of how to induce net transport of passive particles, which are insensitive to the applied drives and/or the geometry of the substrate. A simple technique [3] consists in employing auxiliary A particles that i) interact with the passive species (the B particles) and ii) are easily driven by tunable external forces. By driving such active A particles, one can regulate the motion of the target $\mathrm{B}$ particles through the nano-device of interest.

We discuss here an important property of interacting particles, i.e. their tendency to form clusters. This sure can happen when the pair interaction - between particles of the same or different species - is attractive. Moreover, at low temperatures the attracting particles tend to randomly condense in the wells of the substrate, if any; the substrate translational symmetry is thus broken and the particle mobility markedly affected [4].

A still different mechanism takes place when the interaction between active and passive particles is repulsive: as the active A particles are pulled in one direction by a dc drive, they push along a number of $\mathrm{B}$ particles, of course, at lower speed; the resulting pile-up of particles behaves like a long-lived cluster as long as the dc drive is on. On increasing the dc drive, all clusters involving both $\mathrm{A}$ and $\mathrm{B}$ particles eventually dissolve, the relevant fragmentation thresholds strongly depending on the cluster size and the strength of their mutual interaction. When the particle clusters break up, the A particles continue moving, since they are dcdriven by the external force. More specifically [3], the mobility of the A particles rises to 
one (non-interacting active particle), whereas the mobility of the B particles decays to zero (non-interacting passive particle).

The effectiveness of this type of directed transport can be quantified in terms of the appropriate diffusion coefficients [5]. The diffusion of a single damped Brownian particle is always suppressed in the presence of a periodic substrate potential [6]. However, when the (discrete) mirror symmetry of the periodic substrate is broken, say, by a tilting force, the diffusion constant of the particle can grow much larger than in the absence of a substrate $[7,8]$. Such an excess diffusion is associated with the locked-to-running (or pin-depinning) particle transitions. An exact analytical expression for the excess diffusion coefficient of an overdamped Brownian particle in a tilted periodic potential was derived for arbitrary potentials and noise intensity in refs. [8]. The authors of ref. [8] noticed that in the regime of low temperatures the ratio excess-to-free diffusion constant can be so large that one can talk of a "giant enhancement" of the threshold diffusion.

The central question we address here is whether a similar giant enhancement of normal diffusion can also be observed in the transport of binary mixtures made of active and passive particles or, equivalently, whether the existence of cluster thresholds suffices to originate excess diffusion in the absence of a pinning substrate.

Model. - Let us consider a simple one-dimensional system where $N_{A}$ particles of an active species $\mathrm{A}$ are driven in one direction, say to the right, at a constant speed against penetrable obstacles/traps modelled by movable particles of a second species B, which are not subject to the applied dc drive. We denote by $N_{A}: N_{B}$ the density ration or composition ratio of such a binary mixture. Both $\mathrm{A}$ and $\mathrm{B}$ particles move randomly in a viscous environment kicked around by a zero-mean white Gaussian noise. The A-B interaction is represented by the simplified piecewise linear potential

$$
U_{A B}=\frac{g_{A B}}{\lambda}\left(1-\frac{\left|x_{A}-x_{B}\right|}{\lambda}\right)
$$

for $\left|x_{A}-x_{B}\right|<\lambda$, and $U_{A B}=0$ otherwise. In the overdamped regime the motion of the $\mathrm{A}$ particles is described by the Langevin equations (LE)

$$
\dot{x}_{A, i}=A-\sum_{k} \frac{\partial U_{A A}}{\partial x_{A, i}}\left(x_{A, i}-x_{A, k}\right)-\sum_{j} \frac{\partial U_{A B}}{\partial x_{A, i}}\left(x_{A, i}-x_{B, j}\right)+\xi_{A, i}(t),
$$

where $i, k=1,2, \ldots N_{A}$ label the A particles, $j=1,2, \ldots N_{B}$ labels the B particles, $A$ is a dc drive, and $\xi_{A, i}(t)$ is the random noise acting upon the $i$-th A particle. We further impose that $\left\langle\xi_{A, i}(t)\right\rangle=0$ and $\left\langle\xi_{A, i}(t) \xi_{A, k}(0)\right\rangle=2 T \delta_{i, k} \delta(t)$ (i.e., the Boltzmann constant and the masses have been set equal to one). For the A-A pair interaction we choose the same potential as in eq. (1), with identical interaction length $\lambda$, but tunable strength $g_{A A} \geq 0$ (repulsive). The B particles obey a set of LE like eq. (2), but with interchanged A, B labels and zero drive, namely

$$
\dot{x}_{B, i}=-\sum_{k} \frac{\partial U_{B B}}{\partial x_{B, i}}\left(x_{B, i}-x_{B, k}\right)-\sum_{j} \frac{\partial U_{A B}}{\partial x_{B, i}}\left(x_{A, j}-x_{B, i}\right)+\xi_{B, i}(t) .
$$

In the following, we assume, for simplicity, that $U_{B B}$ and $U_{A A}$ coincide, i.e., $g_{A A}=g_{B B}$.

In refs. $[3,4]$ we developed a perturbative nonlinear Fokker-Planck scheme to treat the coupled dynamics (2)-(3). Here, we limit ourselves to integrate numerically these two sets of LE and to interpret our numerical results within the more conventional framework of the Brownian motion theory [9]. We assume periodic boundary conditions over a unit cell of length 


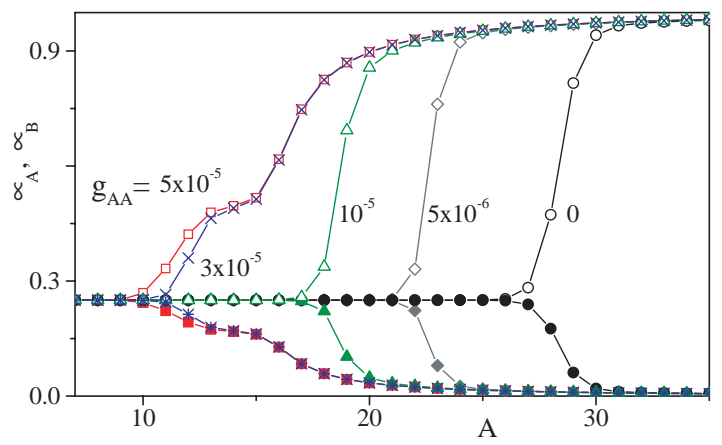

Fig. 1 - (Color online) Mobilities $\mu_{A}$ and $\mu_{B}$ vs. the dc drive $A$ in a $1: 3$ binary mixture of repulsive particles with $g_{A B}=0.02$ and different $g_{A A}=g_{B B}$ (displayed next to each data set). The first fragmentation steps move to the right on decreasing $g_{A A}$. Other simulation parameters: $T=0.005, \lambda=0.05$, and $L=2$.

$L$ containing $N_{A}$ active and $N_{B}$ passive particles, respectively. The quantities we determine to describe the particle transport in the stationary regime are: i) the mobilities $\mu_{A} \equiv\left\langle\dot{x}_{A}\right\rangle / A$ and $\mu_{B} \equiv\left\langle\dot{x}_{B}\right\rangle / A$ of the two species, shown in figs. 1 and 2 . In the absence of external substrates these quantities satisfy the equality $\mu_{A}+N_{B} \mu_{B} / N_{A}=1$; ii) the diffusion matrix $D_{a b}$, with $a, b=A, B$, defined by the normal diffusion law $\left\langle\left[x_{a}(t)-\left\langle x_{a}(t)\right\rangle\right]\left[x_{b}(t)-\left\langle x_{b}(t)\right\rangle\right]\right\rangle=2 D_{a b} t$, shown in figs. 3 and 4 . Here $\langle\ldots\rangle$ implies both a stochastic and an ensemble average; in the absence of particle-particle interaction, the diffusion constant is $D_{0}=T$ for both species.

An additional symmetry of the LEs (2) and (3) is noteworthy. Consider two $N: M$ and $M: N$ mixtures; by means of simple Galileian transformations one proves that $\mu_{A}(M, N)=$ $1-\mu_{B}(N, M)$ and $D_{A A}(M, N)=D_{B B}(N, M)$, as shown in the inset of fig. 4(a). We recall that $N: M$ denotes a mixture with $x N$ A-particles and $x M$ B-particles, for any value of $x$. For this reason we restricted our numerical simulation to mixtures with $N_{A} \leq N_{B}$.

Mobility curves. - In fig. 1 we show the mobility curves $\mu_{A}$ and $\mu_{B}$ vs. the driving force $A$ (applied only to the A particles) for $1: 3$ mixtures of repulsive particles at low temperatures $T \ll\left|g_{A B}\right| / \lambda$ and different values of $g_{A A}=g_{B B} \geq 0$. Results for a $1: 1$ mixture are reported in fig. 2(a).

All mobility curves, no matter what $g_{A A}$, bridge two horizontal asymptotes for small and large $A$, respectively. For large $A$ the drive on the active particles can be so strong that their motion is hardly hampered by the presence of the $\mathrm{B}$ particles; hence $\mu_{A}=1$ and $\mu_{B}=0$. Vice versa for weak dc drives, the A particles are pushed against the B particles, so that all $N_{A}+N_{B}$ particle piles up with mobility

$$
\mu_{A}=\mu_{B}=\frac{N_{A}}{N_{A}+N_{B}} \equiv \mu_{c}
$$

The interaction between pairs of the same species greatly influences the mixture mobilities. For $g_{A A}=0$ both mobilities exhibit one sharp transition jump, from $\mu_{c}$ to 1 for $\mu_{A}$ and from $\mu_{c}$ to 0 for $\mu_{B}$. The mobility jumps for $g_{A A}=0$ correspond to the highest threshold, $A_{c}^{(0)}\left(N_{A}, N_{B}\right)$, observed in a $N_{A}: N_{B}$ mixture; its numerical value is closely reproduced by the zero-temperature analytical estimate of ref. [3], i.e.,

$$
A_{c}^{(0)}\left(N_{A}, N_{B}\right)=\left(N_{A}+N_{B}\right) A_{0}
$$




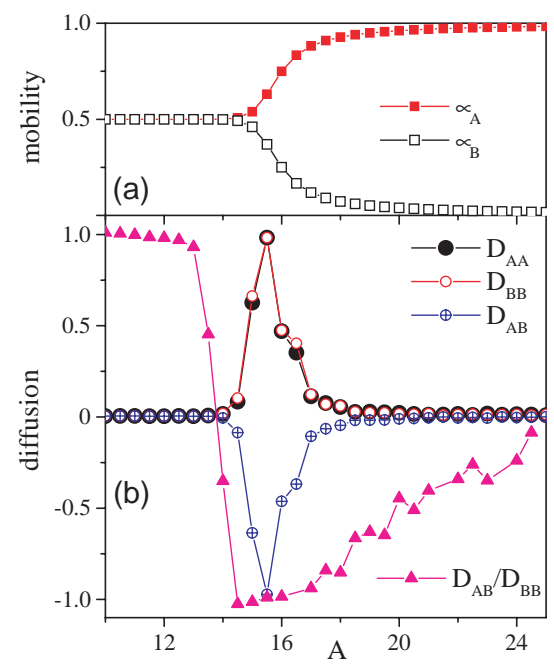

Fig. 2 - (Color online) Transport in a $1: 1$ mixture of repulsive particles: (a) mobilities $\mu_{A}$ and $\mu_{B}$ vs. $A$; (b) diffusion coefficients $D_{a b}$, with $a, b=A, B$, and $D_{A B} / D_{A A}$ vs. A. Other simulation parameters: $T=0.005, g_{A B}=0.02, \lambda=0.05$, and $L=2$.

with $A_{0}=\left|\max \left[\partial U_{A B}(x) / \partial x\right]\right|=g_{A B} / \lambda^{2}$. On increasing $g_{A A}$, the threshold $A_{C}$ shifts towards smaller values with a lower bound $A_{c}^{(\infty)}$ that can be determined as follows. Suppose that A and B particles are entrained in a cluster of size $\left(N_{A}, N_{B}\right)$ with $N_{A}$ active particles (crowed in the left part of the cluster) pushing the $N_{B}$ passive particles to the right. Such an aggregate is stable at $T=0$ as long as the repulsive force between one A, B pair is strong enough to push all the $N_{B}$ passive particles with speed $\mu_{c} A$, namely for $A$ smaller than

$$
A_{c}^{(\infty)}\left(N_{A}, N_{B}\right)=\left(\frac{1}{N_{A}}+\frac{1}{N_{B}}\right) A_{0}=\frac{A_{c}^{(0)}\left(N_{A}, N_{B}\right)}{N_{A} N_{B}} .
$$

The maximum value of $A_{c}^{(\infty)}$ is $2 A_{0}$ and corresponds to the fragmentation threshold of a $(1,1)$ cluster, or dimer.

For large $g_{A A}$ and intermediate $A$, i.e., for $A_{c}^{(\infty)}<A<2 A_{0}$, the mobility curves exhibit a step structure that we relate to the progressive fragmentation of the largest $\left(N_{A}, N_{B}\right)$ cluster into smaller clusters. For instance, in the mixtures of fig. 1, when $A$ increases above $A_{c}^{(\infty)}$, the A particle hops over the first entrained B particle, which in turn drops off the cluster. This results in a new $\left(N_{A}, N_{B}-1\right)$ cluster with larger mobility and higher fragmentation threshold $A_{c}\left(N_{A}, N_{B}-1\right)$, both given by eqs. (4) and (6), respectively, upon replacing $N_{B}$ with $N_{B}-1$. In particular, the prominent $\mu_{A}$ step, from $\frac{1}{2}$ up to close to 1 , at around $2 A_{0} \approx 16$, corresponds to the fragmentation of the smallest and most stable $(1,1)$ clusters. Here, $(1,1)$ refers to clusters with one A and one B particles. Note that this simple mechanism of cluster fragmentation applies both for $N_{A}<N_{B}$ and $N_{A}>N_{B}$, under the condition that $g_{A B}>0$. For $N_{A}=N_{B}$ all clusters resulting from the fragmentation sequence have the same fractional composition of a dimer and, therefore, the same mobility (fig. 2). The mobility steps corresponding to the $(1,3),(1,2)$, and $(1,1)$ clusters are clearly resolved in fig. 1 for a $1: 3$ mixture.

On reversing the sign of $g_{A B}$ the relevant mobility curves retain the step structure discussed above with the caveat that attractive particles tend to form clusters of vanishingly small radius. 


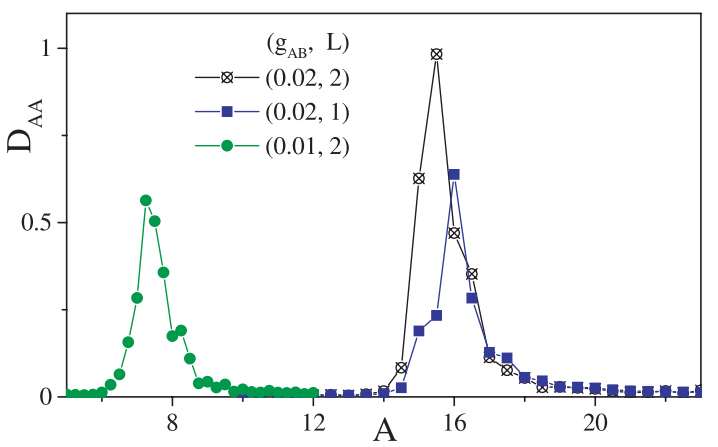

Fig. 3 - (Color online) Excess self-diffusion in a $1: 1$ mixture for different A-B interaction strength $g_{A B}$ and unit cell length $L: D_{A A}$ vs. A. Other simulation parameters: $T=0.005$, and $\lambda=0.05$.

Excess diffusion peaks. - The occurrence of a giant enhancement of the normal diffusion associated with the cluster fragmentation is apparent for $1: 1$ mixtures in figs. 2(b) and 3; and for $1: 3$ mixtures in fig. 4 . The mobility steps are indeed signalled by pronounced peaks in the plots of $D_{A A}$ and $D_{B B}$ vs. A; such diffusion peaks are huge in comparison with the diffusion constant $D_{0}=T$ of a single non-interacting particle. Note that for low drives $D_{A A}, D_{B B} \rightarrow D_{0} /\left(N_{A}+N_{B}\right)$, whereas for large drives $D_{A A}, D_{B B} \rightarrow D_{0}$.

We give now an upper bound for the maxima of the self-diffusion peaks in a $1: 1$ mixture - for which the curves $D_{A A}(A)$ and $D_{B B}(A)$ coincide, as predicted. At about $A_{c}=2 A_{0} \approx 16$, see eq. (6), an active particle switches between a $(1,1)$ bound state and the free running state with drift speed $\frac{1}{2} A_{c}$ and $A_{c}$, respectively. The self-diffusion constant $D_{A A}$ attains its maximum under the condition that the moving A particle in average i) spends the same time in either state (symmetric switches), and ii) completes a full switching cycle from the cluster to the free state and back to the cluster state over one unit cell $L$ (optimal density). During this process its speed increases/decreases symmetrically by the fixed amount $A_{c} / 4$, so that $D_{A A}=(\tau / 2)\left(A_{c} / 4\right)^{2}$ with $\tau=(3 / 2)\left(L / A_{c}\right)$ or, equivalently, $D_{A A}=(3 / 32) A_{0} L$. This estimate for the maximum height of the $D_{A A}(A)$ peaks is in fairly close agreement with the numerical data of figs. 2(b) and 3. By the same line of reasoning one can estimate the height of the $D_{A A}(A)$ peaks for the $1: 3$ mixtures of fig. 4. A similar diffusive behavior was observed also on reversing the sign of $g_{A B}$ (not shown).

For mixtures with $N_{B}>N_{A}$ the self-diffusion of the passive particles, $D_{B B}$, is typically smaller than $D_{A A}$; this is due to the fact that the loose B particles undergo free diffusion with constant $D_{0}$, while the clustered B particles contribute to the self-diffusion an amount equal to $D_{A A}$ each. Let us consider, for instance, the most pronounced diffusion peak in fig. 4; it corresponds to the dimer fragmentation and, therefore, $D_{A A}$ is exceedingly large with respect to $D_{0}$; the ensuing estimate $D_{B B} \simeq\left(N_{A} / N_{B}\right)^{2} D_{A A}$ seems to be in good agreement with our simulation results.

$A-B$ cross-correlation. - The diagonal coefficient $D_{A B}$ of the diffusion matrix is plotted in figs. 2(b) and 4(b), (c). A few remarkable properties are apparent by inspection: i) Below the first fragmentation threshold, the coefficient $D_{A B}$ coincides with $D_{A A}$ (and $D_{B B}$ ), as all $\mathrm{A}$ and $\mathrm{B}$ particles are clumped together; ii) On increasing $A$ close to such a fragmentation threshold (how close it depends on $T$ ), $D_{A B} / D_{A A}$ drops from 1 down to negative values (-1 for the $1: 1$ mixtures of fig. 3 , and $-\frac{1}{3}$ for the $1: 3$ mixture of fig. 4 ), which it then retains over a wide $A$ interval; iii) For $A \gg A_{0}$, namely for dc drives well above the dimer fragmentation threshold, $D_{A B}$ tends to zero from negative values, seemingly according to a power law (in 


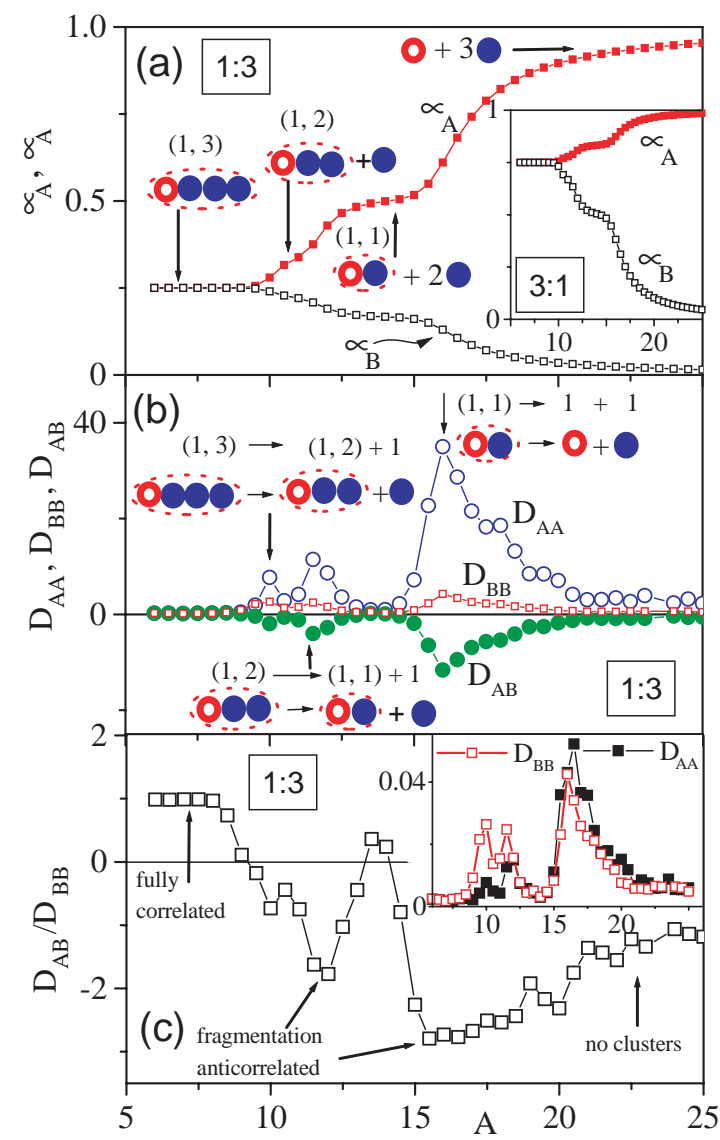

Fig. 4 - (Color online) Excess diffusion and mobility in $1: 3$ and $3: 1$ mixtures. (a) Mobility $\mu_{A}$ and $\mu_{B}$ vs. $A$ for a $1: 3$ (main panel) and $3: 1$ mixture (inset). $\mu_{A}$ is the upper (red) curve and $\mu_{B}$ is the lower (black) curve. Plateaus correspond to stable clusters (vertical arrows); (b) Diffusion $D_{A A}$ (blue-open circles), $D_{B B}$ (red-open squares), and $D_{A B}$ (green-solid circles) vs. $A$ for the $1: 3$ mixture. Peaks signal cluster fragmentation (vertical arrows). Schematic diagram for clusters and cluster fragmentation in the $1: 3$ mixture are represented in (a) and (b) by means of red-open (A particle) and blue-solid circles (B particles). (c) $D_{A B} / D_{B B}$ vs. $A$ for the $1: 3$ mixture. Note that $(1,3)$ clusters result in fully correlated particle motion, $D_{A B} / D_{B B} \approx 1$; cluster fragmentation corresponds to strongly anti-correlated diffusion of A's and B's. Inset in (c): $D_{A A}$ and $D_{B B}$ vs. $A$ for the $3: 1$ and the $1: 3$ mixture, respectively; simulation data points tend to coincide (see text). Other simulation parameters: $T=0.005, \lambda=0.05, g_{A B}=0.02, g_{A A}=g_{B B}=5 \times 10^{-5}$, and $L=2$.

the same limit, $\left.D_{A A} \rightarrow 0+\right)$. It follows that, at fragmentation, the diffusion of the A and B particles is markedly anti-correlated.

The explanation of the properties i)-iii) above for a $1: 1$ mixture is straightforward. Let us consider the variance of the A-B distance, $\left\langle\left[x_{A}(t)-x_{B}(t)\right]^{2}\right\rangle-\left[\left\langle x_{A}(t)\right\rangle-\left\langle x_{B}(t)\right\rangle\right]^{2}=2 D_{A-B} t$; the coefficient $D_{A-B}$ is related to the coefficients of the diffusion matrix by $D_{A-B}=2\left(D_{A A}+\right.$ $D_{A B}$ ) (note that here $\left.D_{A A}=D_{B B}\right)$; hence,

$$
\frac{D_{A B}}{D_{A A}}=1-\frac{D_{A-B}}{2 D_{A A}} .
$$


Under the conditions itemized above: i) for a cluster, $D_{A-B}=0$ yields $D_{A B} / D_{A A}=1$; ii) the fragmentation onset, $x_{B}(t)-\left\langle x_{B}(t)\right\rangle \doteq-\left[x_{A}(t)-\left\langle x_{A}(t)\right\rangle\right]$ (here, $\doteq$ means statistical equivalence), corresponds to $D_{A-B}=4 D_{A A}$, i.e., $D_{A B} / D_{A A}=-1$; iii) finally, for large drives, the limiting values $D_{A A}=D_{0}$ and $D_{A-B}=2 D_{0}$ imply a vanishing $D_{A B} / D_{A A}$ ratio.

Based on the previous argument, we conclude that the negative $D_{A B} / D_{A A}$ plateau of a $1: \mathrm{N}_{B}$ mixture is to be expected at around $-1 / N_{B}$ (not shown). Figure 4 supports our predictions with a caveat: Away from the fragmentation thresholds, no anti-correlation is expected, so that $D_{A B} / D_{A A}$ is dominated by the diffusion of the bound A and B particles. This effect becomes visible in fig. $4(\mathrm{c})$, where the $(1,1)$ cluster region is signalled by a reentrant positive peak of $D_{A B} / D_{A A}$.

In this letter we have shown that giant diffusion in one-dimensional mass transport can originate from the fragmentation of clusters or other aggregates of driven interacting particles, with no regard to the (possibly concurrent) pinning action of the substrate. Recent advances in nanotechnology $[10,11]$ provide an ideal playground for the study of diffusion in restricted geometries; prominent examples, among others [11], are colloids trapped in light channels [12], solutes of different mass in asymmetric silicon pores [13], and superconducting devices supporting two distinctly controllable vortex species [14]. Extensions could also apply to systems driven by several frequencies [15].

\section{REFERENCES}

[1] Alberts B. et al., Molecular Biology of the Cell (Garland, New York) 1994; Morais-CabraL J. H. et al., Nature (London), 414 (2001) 37.

[2] Kärger J. and Ruthven D. M., Diffusion in Zeolites and Other Microporous Solids (Wiley, New York) 1992.

[3] Savel'ev S., Marchesoni F. and Nori F., Phys. Rev. Lett., 92 (2004) 160602; 91 (2003) 010601.

[4] Savel'ev S., Marchesoni F. and Nori F., Phys. Rev. E, 71 (2005) 011107; 70 (2004) 061107.

[5] Machura L. et al., Phys. Rev. E, 70 (2004) 061105; Machura L. et al., J. Phys. Condens. Matter, 17 (2005) S3741.

[6] Lifson S. and Jackson J. L., J. Chem. Phys., 36 (1962) 2410; Festa R. and D'Agliano E. G., Physica A, 90 (1978) 229.

[7] Costantini C. and Marchesoni F., Europhys. Lett., 48 (1999) 491.

[8] Reimann P. et al., Phys. Rev. Lett., 87 (2001) 010602; Phys. Rev. E, 65 (2002) 031104.

[9] Risken H., The Fokker-Planck Equation (Springer, Berlin) 1984, Chapt. 11.

[10] For reviews see: the special issue in Appl. Phys. A, 75 (2002) 167 on Ratchets and Brownian Motors: Basics, Experiments and Applications, edited by Linke H.; and the issue in Chem. Phys., 281 (2002) 111 on Transport in Molecular Wires, edited by HÄNGGi P., RATnER M. and YALIRAKI S.

[11] Hänggi P., Marchesoni F. and Nori F., Ann. Phys. (Leipzig), 14 (2005) 51.

[12] Korda P. T., Taylor M. B. and Grier D. G., Phys. Rev. Lett., 89 (2002) 128301; Lutz C. et al., Phys. Rev. Lett., 93 (2004) 026001; Wei Q.-H., Bechinger C. and Leiderer P., Science, 287 (2000) 625; Mangold K., Leiderer P. and Bechinger C., Phys. Rev. Lett., 90 (2003) 158302; Bubeck R., Leiderer P. and Bechinger C., Europhys. Lett., 60 (2002) 474.

[13] Matthias S. and Muller F., Nature (London), 424 (2003) 53; Kettner C. et al., Phys. Rev. E, 61 (2000) 312.

[14] Villegas J. E. et al., Science, 302 (2003) 1188; Savel'ev S. and Nori F., Nature Mater., 1 (2002) 179; Savel'ev S. and Nori F., to be published in Physica C; Cole D. et al., Physica C, 404 (2004) 99.

[15] Savel'ev S., Marchesoni F., Hänggi P. and Nori F., Europhys. Lett., 67 (2004) 179; Phys. Rev. E, 70 (2004) 066109; European Phys. J. B, 40 (2004) 403. 